\title{
Nonlinear Processing at Signal Conditioning of Gas Sensors Based on Semiconductor Nanocomposites
}

\author{
B.O. Semerjyan \\ Center of Semiconductor Devices and Nanotechnologies, \\ Yerevan State University, 1 Alex Manoogian Street, 0025 Yerevan, Armenia \\ E-mail: semicsemer@ysu.am
}

Received: December 1, 2021; Revised: December 17, 2021; Accepted: December 25, 2021

\begin{abstract}
One of the major advantages of high resistance gas sensors (HRGS) based on MWCNT/SnO ${ }_{2}$ nanocomposite is the relatively high variation of the output parameter. However, these types of GSs have several specific features making the processing of gas registration and measurement results difficult. These features involve distribution of resistance at very low registration values of gas concentration, and considerable non-linearity of load characteristic as a result of inversely proportional character of transformation function from gas concentration to resistance. Elimination of these unfavorable characteristics is possible also to perform in input measurement circuits through proportional changing of HRGS conductivity to electric signal. For linearization of output characteristics of $\mathrm{MWCNT} / \mathrm{SnO}_{2}$ gas sensors, we propose also to use the Wheatstone bridge circuit.
\end{abstract}

Keywords: gas sensors, nonlinearity, Wheatstone bridge, linearization scheme

https://doi.org/10.54503/18291171-2021.14.4-191

\section{Introduction}

Semiconductor $\mathrm{SnO}_{2}$ gas sensors (GS) and their signal conditioning systems currently are of a special interest $[1,2]$. The GSs are highly sensitive, small of size, and have a low cost. For activation of the sensor gas sensitivity and gas desorption from a sensor it mshould be subjected to heating. The heating procedure makes complicated the application of this type of sensors particularly for control of fuels and flammable gases. Decrease of the temperature of the maximal gas sensitivity is an important issue. For improvement of features of gas sensors as devices, some experiments studied gas sensitivity by measurements of volt-amperometric characteristics of gas sensitive thin films [3]. Recent advances in nano-sensor development include practicing of nanomaterials [2].

On the other hand, for practical applications it is also important to achieve the linearization of nonlinear output signal of GS [4]. Due to the advancement of IC technologies, the hardware implementation of the software methods can be done easily with reduced time, cost, and more accuracy. However, due to availability of high performance analog devices, the analog methods are still popular among many researchers [5].

The accuracy of linearization schemes depends on the type and the nonlinearity value of the sensor output. Since it is difficult to find an exact polynomial equation or other functions to represent the response curve, it gives more error when the measurement parameter is determined from the inverse approximation functions. As many sensors are used for different applications, the linearized characteristics will simplify the design, calibration, and accuracy of the measurement. This paper presents an analog method applied to linearize gas sensor (GS) characteristics by a nonlinear processing in signal conditioning [6].

The present research focuses on study of (i) non-linearity characteristics and parameters of electrical, thermo- and gas-sensitivities of gas-sensitive materials and devices and (ii) linearization 
of nonlinear highly- and weakly-resistive gas sensors based on $\mathrm{MWCNT} / \mathrm{SnO}_{2}$ structures with nanocomposites by shunting GS in Wheatstone bridge.

\section{Results and discussions}

\subsection{Electrical sensitivity}

Gas sensitive properties are exhibited by many metal oxides. Among these oxides, the $\mathrm{SnO}_{2}$ is mostly used in gas sensors [1]. This material is well studied and serves as a model material for all metal oxide gas sensors [7].

In studies [8,9], it is demonstrated that the $\mathrm{SnO}_{2}$-based sensors have nonlinear currentvoltage characteristics with a behavior that is dependent on environment conditions. The interpretation of nonlinearity of current-voltage characteristics needs consideration of the two processes: the variation of the barrier height at grain boundaries and tunneling of electrons through surface states.

All factors that influence the sensor characteristics may be classified as reversible (the interruption of the influence of such a type factors results in return of the conductivity to initial level) and nonreversible. Slow reversible and nonreversible processes depend on the prehistory of the sample state. The electrophysical properties of gas sensitive films are influenced by many environmental factors, regime of measurements, etc. [10].

Sensor signal has been considered by volt-ampermetric method. Gas sensor can be characterized by differential electroconductance (differential steepness) and can be calculated as tangent of the tangential angle slope to the volt ampere curve in a given point, considering the axis scale. GS current-voltage characteristic is often taken into account. GS resistance non-linearity coefficient would be as

$$
\gamma=\frac{\sigma_{g}}{\sigma}=\frac{U}{I} \frac{d I}{d U}
$$

For a GS with any linearity $\gamma$ equals to 1 , while nonlinear current-voltage curves it varies from 1. Non-linearity coefficient would be a constant value if the volt ampere curve fits the equation:

$$
I=B U^{\gamma}
$$

if $\gamma=$ const. Current-voltage curve is approximated by equations (2) and (3):

$$
U=A I^{\alpha}
$$

where A, B, $\alpha$ and $\gamma$ are constant values, $\gamma=1 / \alpha$ and $B=A^{-1 / \alpha}$. These formula fit also to narrow range.

Nonlinearity coefficient is calculated as

$$
\gamma=\lg \frac{I_{2}}{I_{1}} / \lg \frac{U_{2}}{U_{1}}
$$

where $I_{2}, I_{1}, U_{2}$, and $U_{1}$ are respectively the measures of currents and voltages chosen for narrow range of values.

The true characteristics of gas-sensitive structures and the characteristics of gas sensors made of these structures differ from equations here above; the nonlinearity coefficients $\alpha$ and $\gamma$ are functions of the applied voltage and vary from point to point. This makes the assessment of GSs quality as well as their comparison rather difficult. 
Ohm law can be inferred from more general formula for current-voltage curve of nonlinear GS resistance in a particular case (see equation (2)), if $\gamma=1$, i. e. consider a resistance of GS with no dependence of its electroconductance on applied voltage.

\subsection{Temperature sensitivity}

The environmental temperature may have a substantial effect on GS characteristics. It can be expressed by the following equations:

$$
R=M e^{\frac{l}{T}}, \quad T C R=\frac{1}{R} \frac{d R}{d T}=-\frac{l}{T^{2}},
$$

where $M$, and $l$ are constant values that characterize the resistance at a special voltage, TCR is the temperature coefficient of the resistance.

For more convenient practical purposes one could use the temperature coefficient of the current variation at different values of the applied voltage:

$$
T C I=\frac{1}{I} \frac{d I}{d T} .
$$

Conductance thermodependence studies can reflect intrinsic physical processes in sensor performance, since it is an essential characteristic of a sensor as a device [11]. Gas sensor conductance thermodependence relies on both the environment conditions and the sensitive layer parameters such as doping [12]. Conductance thermodependance in humid atmosphere is related also to maximum temperature of the preceded heating.

For some types of GSs, for conductivity it is typical the anomalous temperature dependence [13]. In contrast to ordinary semiconductors, for which the conductivity is expressed by activation of nonlinear monotonic dependences with different slopes in different temperature ranges, TCI $T$ ), the above mentioned GSs exhibit bell-shaped temperature dependence. Manifestation of such type temperature dependence is a criterion of a high gas sensitivity of $\mathrm{SnO}_{2}$-based GSs [14]. The bellshaped temperature dependence is typical of reducing gases [15].

In $\mathrm{Ar}$ atmosphere, the temperature dependence of conductivity of $\mathrm{SnO}_{2}$ thin films exhibit exponential dependence of the conductivity on inverse temperature, with activation energy of 0.72 $\mathrm{eV}[16]$. In study $[17,18]$, it is proposed a process for fabrication of chemical sensing employing multi-walled carbon nanotubes (MWNTs) as the active sensing element. The studies [19] reported on investigation of gas sensing characteristics of $\mathrm{MWNT}_{\mathrm{SnO}} \mathrm{Sn}_{2}$ nanostructures. Among $\mathrm{MWNT} / \mathrm{SnO}_{2}$ nanostructures, the best samples are characterized by a high and selective response with respect to butanol vapors in the temperature range of $150-200^{\circ} \mathrm{C}$.

\subsection{Gas sensitivity}

This research focuses on alterations of current intensity in a circuit with a gas sensor (GS) related to variations of partial pressure of the surrounding registered gas in oxygenated atmosphere.

Testing of decorated semiconductor nanocomposite-based gas-sensitive high-ohmic samples applied in instrument for detection of partial gas pressure (PGP) or related to volume concentration of this gas, $\mathrm{C}$ (ppm), in oxygenated atmosphere includes the analysis of the working range and coefficient of transformation (sensitivity).

Let's assume that GS resistance varies proportionally to concentration of the registered admixture gas in atmosphere. The total baseline GS resistance is $R_{0} ; \rho$ is the resistivity for variation per unit concentration of analit gas; $I_{0}=\varepsilon / R_{0}$ is the baseline current intensity. Increase of the 
registered gas concentration by $\Delta C$ will result in increment of the GS current intensity $\Delta I$ provided the condition $\frac{\rho}{R_{0}} \Delta C \ll 1$ is fulfilled:

$$
\triangle I=\frac{\varepsilon}{R_{0}}-\frac{\varepsilon}{R_{0}-\rho \Delta C} \approx I_{o} \frac{\rho}{R_{0}} \Delta C
$$

The increase of the registered gas concentration by $\Delta C$ will increase the current by $\Delta I=$ $S_{I} \Delta C$, where $S_{I}=I_{0} \frac{\rho}{R_{0}}$. $S_{I}$ is another essential GS parameter that is defined as the "coefficient of transformation". Hence, it follows from equation (7) that for a registered small variation of the gas concentration in the air, $\Delta C$, the variation of the current intensity $\triangle I$ is directly proportional to $\Delta C$.

The sensor with output signal proportional to input disturbance is known as linear. Linearity in working range is an essential characteristic of a sensor. It defines the GS range of registered gas volume concentrations. The nonlinearity of a gas sensor possibly can be reduced by means of circuit's methods rather than constructive methods (technologies). For example, sensor nonlinearity could be decreased by a potentiometric circuit with sequential to GS turnover of the indicator. In this pattern of circuit the non-linearity of the GS current dependence upon concentration of the registered gas $I(C)$ is much less compared to the dependence upon pattern $R(C)$.

Further improvements of gas sensors suggest increase of linearity and precision of the measurements. The sensitivity improvement is obtained also by increase of the transmission characteristic $S_{I}$ (e.g. at expense of EMF of battery $\varepsilon$ ).

The detection limit of the chemical species registered by the sensors such as metal oxide, thermoelectric and other type gas sensors are affected by the nonlinear response. Electrical response $R_{C}$ of the metal oxide gas sensor for reducing gas with the variation of gas concentration $(C)$ can be represented by an empirical relation $[4,18]$ :

$$
R_{C}=k C^{-\delta}
$$

where $k$ is the characteristic coefficient of the gas sensing film, and $\delta$ is the slope of the response curve. For oxidizing gas, the resistance value increases with increase in gas concentration [20].

The influence of the signal (dynamic and static) amplitude variations measurements on performance of potentiometric and bridge circuit are in the following.

The potentiometric circuit is more suitable for measurements of dynamic variations of gas concentrations. It allows general earthen of both GS and detection equipment. The output signal is connected through a capacitor or another filter for elimination of the constant component of the signal. Apparent signal amplitude variation or the zero drift to the temperature is often of low frequency and is excluded by the filtration mentioned.

Appliance of series resistance exceeding the one of GS, the efficiency of the circuit may reach 90\%. Therefore, a stabilized constant current source can be applied for the GS. Additionally, thermosensitivity of variations with temperature can be decreased. Such preconditioning allows output signal variation with temperature to set as variation with temperature as $\Delta R$ rather than $\Delta \mathrm{R} / \mathrm{R}$.

Static measurements of gas concentrations require a bridge circuit. In more simple conditions that ignore high precision measurements and maintain a relatively constant temperature, the sensor resistance can be measured via the Wheatstone bridge. These measurements are linear for registration of small concentrations of the gas of interest.

For identical arms (i.e., all he resistances) non-linearity may reach $10 \%$ if active arm resistance $R_{1}$ increases by $20 \%$. The non-linearity is reduced when $R_{2}$ and $R_{4}$ are significantly higher than $R_{1}$ and $R_{3}$. To compensate the temperature of the zero drift, $R_{3}$ should be replaced with an idle sensor (Fig. 1).

Three types of circuits are used in resistive gas sensor connections: 
1. Serial chain - the GS is connected with powering voltage $\varepsilon$ and load resistance $\left(R_{L}\right)$ in serial pattern;

2. Voltage divider - the GS connected parallel to load resistance. $R_{1}$ is resistance serially connected to GS in the chain;

3. Bridge circuits.

First two connections present non-linear correlation between $R_{c}$ and $U_{\text {out }}$. Particularly, in the energizing of the circuit the EMF $\varepsilon$ is for the voltage divider,

$$
U_{\text {out }}=\varepsilon R_{c} R_{L} /\left[\left(R_{C} R_{L}+R_{1}\left(R_{C}+R_{L}\right)\right]\right.
$$

This infers that even the open circuit operation $\left(R_{L} \gg R_{c}\right)$ do not alter its transferring characteristic:

$$
U_{\text {out }}=\varepsilon \frac{R_{C}}{R_{C}+R_{1}}
$$

This correlation should be linear for reduction of additional transformations as well as providing constant sensor sensitivity through whole range of transformed value. That can be achieved either when GS operates within limited zone of transferring characteristic or circuit powering from constant current source $\left(I_{o}=\right.$ const). In the latter case, when $R_{1} \gg R_{C}$, then $U_{\text {out }}=$ $I_{o} R_{c}$.

A common drawback of potentiometric circuits is the non-zero output signal. A small value of the valid signal is measured in the background of high symphasic disturbance. This drawback is prevented by bridge circuit.

Registration by low-resistive semiconductor GS are performed primarily by balanced bridge circuits. The benefit of the method is its high precision and appliance in minor variations of GS resistance.

Registration with high-resistive GS may develop significant variations of GS resistance. Therefore, balanced bridge circuits are hard to practice. While in the unbalanced bridge,detection of $\Delta R / R$ value relevant to the current or resistance magnitudes in bridge detection diagonal is much simpler.

\subsection{Gas sensor shunt effect on its output characteristic}

The shunting of the HRGS resistance $R$ by a constant resistance $R_{\text {sh }}$ leads to a significant change in loading characteristics as $R=f(C)$ (Fig. 1). The index of shunting (shunt characteristic) is a ratio $g=R / R_{s h}$, which is determined at $R=R_{0}$. Equivalent resistance of two-terminal network "HRGS-Shunt" is $R_{1}^{1}=R R_{\text {sh }} /\left(R+R_{s h}\right)$. At absence of a HRGS gas environment $R_{s h} \ll R$, and then $R_{1}^{1} \approx R_{S h}$. The latter means that the two-terminal network resistance with HRGS in air environment without registered gas is determined by the shunt resistance. Consequently, HRGS shunting make it possible to eliminate uncertainty of the GS resistance at gas zero concentration. The major disadvantage of shunting is the decrease of HRGS sensitivity. The relative variation of sensitivity $S$ is detected at $C_{\text {nom }}$ (nominal concentration of detected gas):

$$
S=\frac{\Delta R_{1} / R_{1}}{\Delta R / R} 100 \%
$$

where $\Delta R_{1}=R_{1}-R_{1}^{1} ; R_{1}^{1}$ is the two-terminal network resistance with HRGS- $\mathrm{R}_{\mathrm{sh}}$ in air environment with registered gas. Let us introduce the following notations:

$$
R_{s h}=R / g ; R_{1}=R /(1+g) \text { and } S_{R}=\Delta R / R,
$$


where $S_{R}$ is the relative variation of HRGS resistance. It is obtained that:

$$
R_{1}^{1}=(R-\Delta R) R /[R+g(R-\Delta R)]=\left(1-S_{R}\right) \mathrm{R} /\left[1+g\left(1-S_{R}\right)\right]
$$

where

$$
\Delta R_{1}=R \Delta R /[R+g(R-\Delta R)](1+g)=R S_{R} /\left[1+g\left(1-S_{R}\right)\right](1+g)
$$

and

$$
\begin{aligned}
& S_{R 1}=\frac{\Delta R_{1}}{R_{1}}=\frac{\Delta R}{R+g(R-\Delta R)} 100 \%, \\
& S=R 100 \% /[R+g(R-\Delta R)]=100 \% /\left[1+g\left(1-S_{R}\right)\right] .
\end{aligned}
$$

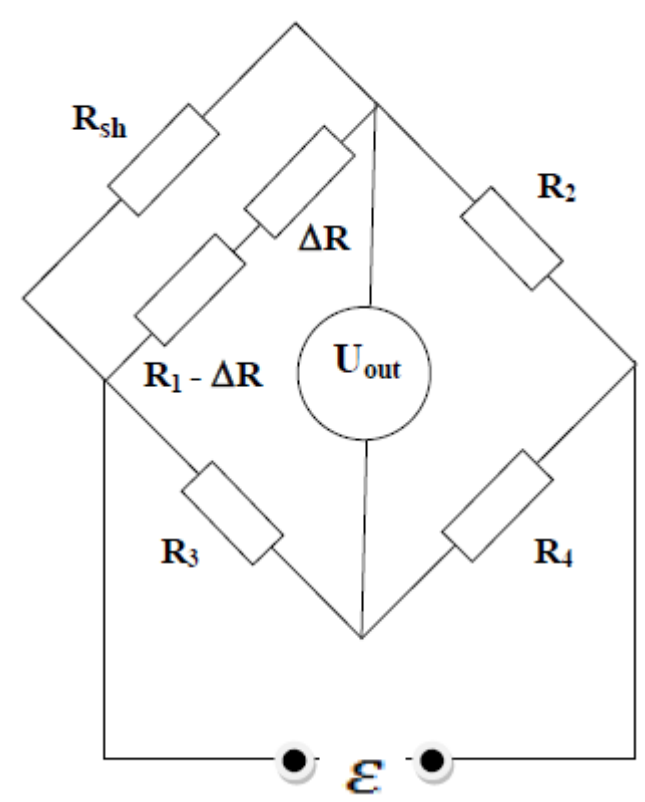

Fig. 1. The Wheatstone bridge circuit with two-terminal network (gas sensor $R_{1}$ and $R_{\mathrm{sh}}$ ) and other bridge resistances $R_{2}, R_{3}$, and $R_{4}$.

Obviously, along with reduction of the shunt resistance (with increasing $g$ ) the nonlinearity of characteristic is sharply reduced at values $g \leq 5$ (at $g=5$ the nonlinearity decreases twice). Further increase in $g$ will lead to slow decrease of nonlinearity.

GS pattern of performance in Wheatstone bridge circuits is similar with that of semiconductor tenso- and thermo-resistors [6]. Certain calculation methods for parameters of bridge circuits of the latter sensors are applicable also for GS. However, due to higher values of $\Delta R / R_{O}$, the GS bridge circuit calculations are highly dependent on the choice of optimal bypass (shunt) value $R_{\text {sh }}$ that should combine ultimate GS sensitivity and the least non-linearity.

Essential distinguishing feature of nanocomposites-based HRGS [21] is the instability of $R_{0}$ and high values of $\Delta R / R_{0}$ which alter the design method and the parameters of the circuit. Consequently, in Wheatstone bridge circuit design for HRGS considers mainly the optimal value of shunt $R_{s h}$, expressing a high sensitivity at weak nonlinearity of the load characteristic of HRGS.

The output signal voltage $U_{\text {out }}$ in the bridge circuit is determined by expression

$$
U_{\text {out }}=\varepsilon\left(\frac{R_{1}-\Delta R_{1}}{R_{1}-\Delta R_{1}+R_{2}}-\frac{R_{3}}{R_{3}+R_{4}}\right),
$$


where $\varepsilon$ is the bridge supply voltage; $R_{1}$ (GS resistance at no gas exposition), $R_{2}, R_{3}, R_{4}$ are the bridge arms' resistances (Fig. 1).

Consider an example of the Wheatstone bridge parameters circuit design with "HRGS-Shunt" two-terminals included in one of the bridge arms with symmetry relatively to diagonal of power supply, i. e. $R_{1}=R_{3}$ and $R_{2}=R_{4}=m R_{1}$. According to [22] nonlinearity of bridge output characteristic $\mathrm{N}(\mathrm{m})$ is determined from equation:

$$
N(m)=(4+m) \Delta R_{1} 100 \% / 4(1+m) R_{1} .
$$

Substituting expression (15) in (18), it is obtained:

$$
N(m)=(4+m) S_{R} 100 \% / 4(1+m)\left[1+g\left(1-g\left(1-S_{R}\right)\right]\right.
$$

If $R=0.5 G \Omega$ and $\Delta R / R=0.9$, we can construct $N=N(m)$ dependence plot at several values of $g$ (Fig. 2). From received family of curves we can obtain $m$ on assumption of reasonable $N$-value of output characteristic's nonlinearity. Obviously, the higher is the $m$ (at prescribed value of $g$ ), the less is the nonlinearity of the characteristic. However, increase of $m$ above $10 \div 20$ is undesirable since the characteristic nonlinearity $N$ decrease is insignificant, while $\varepsilon$-power supply voltage of the circuit sharply increases. Hence, the choice of $m$ value of the bridge is limited by power supply voltage.

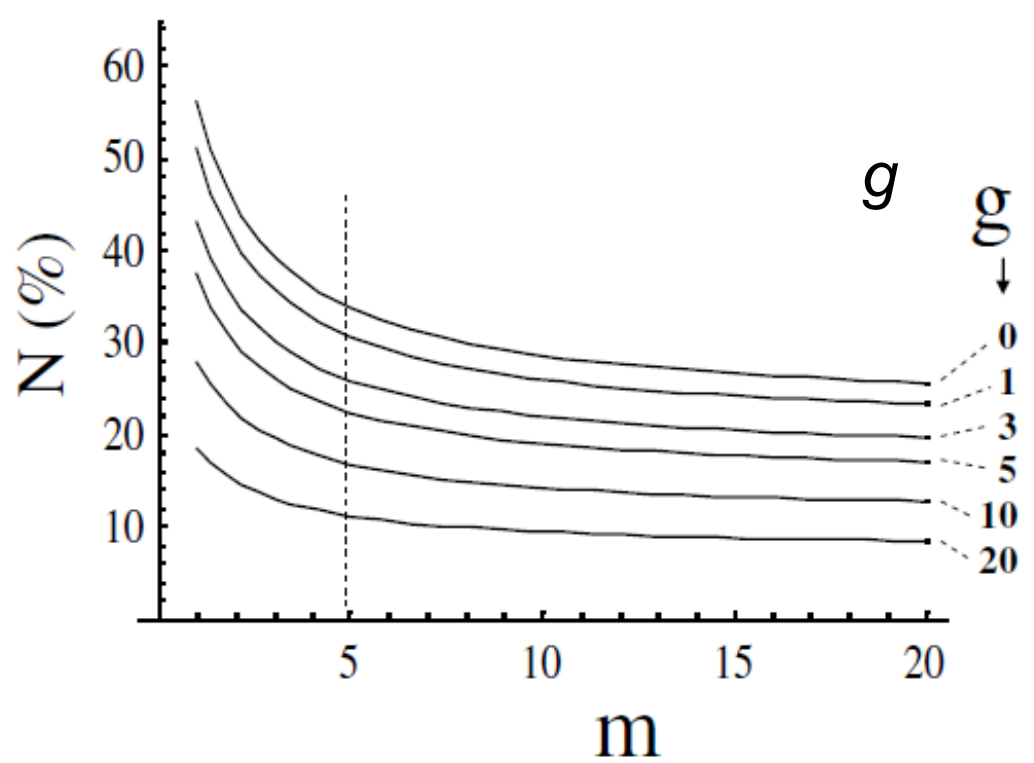

Fig. 2. The dependence of nonlinearity of the bridge circuit output characteristic $N$ on parameter $m$ at different values of $g$.

Determination of power supply voltage of bridge circuit is performed based on HRGS operating current value $I$ :

$$
\varepsilon=I(R-\Delta R)+\left(I+I_{s h}\right) R_{2},
$$

where $I_{s h}$ is the current in shunt. It is obvious that

$$
I_{S h}=\frac{I(R-\Delta R)}{R}=I\left(1-S_{R}\right) g .
$$

Expressing $R_{2}$ by

$$
R_{2}=m R /(1+g)
$$


and making the substitution (21) and (22) into (20), it is obtained that:

$$
\varepsilon=I\left[(\mathrm{R}-\Delta R)+\frac{m R+(R-\Delta R) g m}{1+g}\right]=I R\left\{\left(1-S_{R}\right)+\frac{m\left[1+g\left(1-S_{R}\right)\right]}{1+g}\right\}=I r .
$$

Hence, the bridge power supply voltage is a function of both HRGS operating current and circuit common resistance $r$ depending on bridge parameters, particularly, the coefficients $m$ and $g$. To set the bridge power supply voltage $\left(R=0.5 G \Omega\right.$ and $\left.\Delta R / R=S_{R}=0.9\right)$ we construct dependence $r=r(m)$ at several values of $g$ (Fig. 3). It follows from the graph that along with increase of $m$ there is significant elevation of $r$ as well as the bridge power supply voltage.

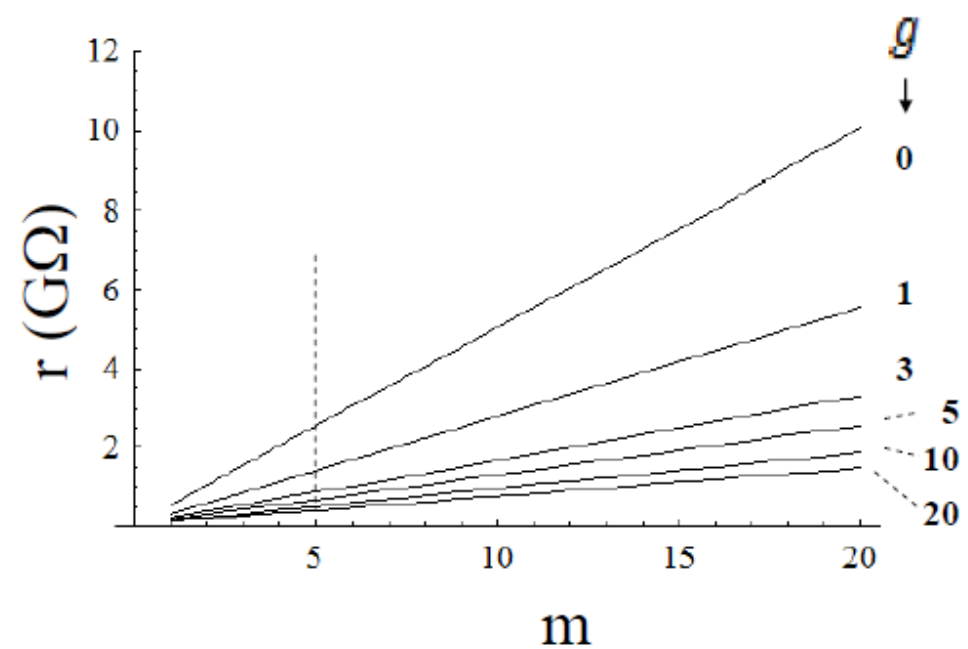

Fig. 3. The dependence of the bridge circuit common resistance $r$ on parameter $m$ at different values of $g$.

The output signal voltage bridge of measuring diagonal at HRGS resistance altered from $R$ to $R-\Delta R$ and the internal resistance of registering voltmeter $R_{l}=\infty$, can be determined from expression (17). After substitution of (12), (13), and (22) into (17), we obtain:

$$
U_{\text {out }}=\frac{\varepsilon m \Delta R}{(m+1) \Delta R-(m+1)^{2}[R+g(R-\Delta R)]}=\varepsilon \frac{m S_{R}}{(m+1) S_{R}-(m+1)^{2}\left[1+g\left(1-S_{R}\right)\right]}=\varepsilon K(m) .
$$

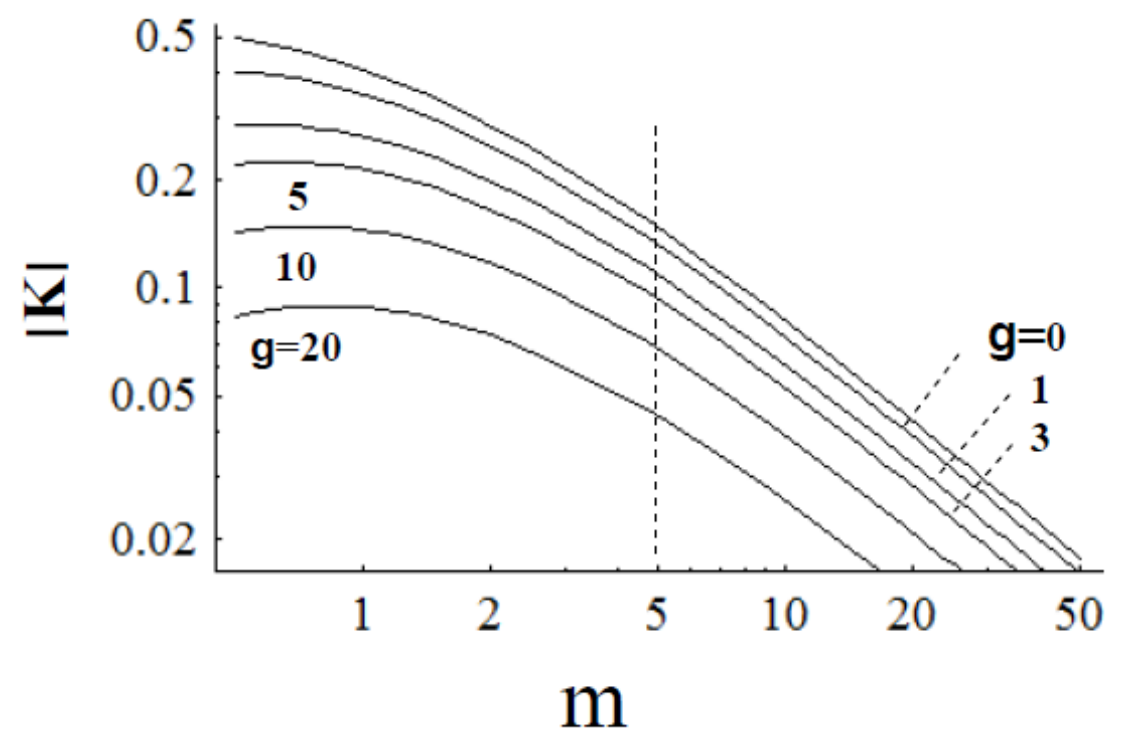

Fig. 4. The dependence of the coefficient $\mathrm{K}$ on parameter $m$ at different values of $g$. 
Analogously with preceding construction $\left(R=0.5 G \Omega\right.$ and $\left.S_{R}=\Delta R / R=0.9\right)$ we plot the dependence $K(\mathrm{~m})$ at several values of $g$ (Fig. 4) and determine expected value of signal voltage. For example, if $m=5, g=5, I=50 \mathrm{nA}$, then $=30 \mathrm{~V}, K=0.093$, and $U_{\text {out }}=2.78 \mathrm{~V}$.

\section{Conclusions}

Nonlinearity feature was studied in three different circuits with various patterns of resistive GS connections. Benefits and drawback of potentiometric versus bridge measurement circuits in dynamic and static variations of registered gas concentrations were shown.

In this study, for linearization of output characteristics of $\mathrm{MWCNT} / \mathrm{SnO}_{2}$ gas sensors, we propose to use the Wheatstone bridge circuit. GS resistance $R$ shunting by constant resistance $R_{\text {sh }}$ leads to significant change in loading characteristics. From analysis of GS shunt effect on its output characteristic, it is obtained that along with reduction of shunt resistance (with increasing g) the nonlinearity of characteristic is sharply reduced at values $g<5$ (at $g=5$ nonlinearity decreases twice. Further increase in $g$ will lead to slow decrease of nonlinearity. Essential distinguishing feature of nanocomposite-based high resistance GS (HRDS) with no use of Wheatstone bridge circuit is the instability of $R_{0}$ and high values of $\Delta R / R_{0}$. Consequently, application of the Wheatstone bridge circuit design for HRGS, at optimal $R_{\text {sh }}$, mainly results in a high sensitivity at a low nonlinearity of the load characteristic.

The theoretical calculations conducted in this study are also applicable to low resistance GSs owing to their "internal shunting". The shunting effect may be produced by doping of the sample during the technological process of preparation of a GS. The Wheatstone bridge characteristics dependent on parameters $g$ and $m$ (the nonlinearity of output characteristic, the common resistance, the power supply voltage of circuit, and the output signal voltage) are calculated with the twoterminal network "HRGS- Shunt" included in one of the bridge arms. We have obtained terms for HRGS performance linearization at preserved sufficiently high gas sensitivity and stability that permit application of standard electronics for registration of bridge readings.

\section{Acknowledgements}

The author wishes to thank Dr. Z. N. Adamyan and Dr. M. S. Aleksanyan for fruitful discussions.

\section{References}

[1] S. Solomon, Sensors Handbook (Mc Graw-Hill, 2010).

[2] V.M. Aroutiounian, Journal of Contemporary Physics (Armenian Academy of Sciences) 50 (2015) 333.

[3] V.V. Simakov, O.V. Yakushev, A.I. Grebennikov, V.V. Kisin, Sensors and Actuators B: Chem. 116 (2006) 221.

[4] J. Fraden, Handbook of Modern Sensors Physics and Applications (Springer, San Diego, 2003).

[5] A.S. Morris, R. Langari, Mesurement and Instrumentation: Theory and Applications (Elsevier, New York, 2012).

[6] L.S. Ilyinsakaya, Semiconductor Tenzosensors (Energiya, Moscow, 1975).

[7] A. Gurlo, Chem. Phys. Chem. 7 (2006) 2041.

[8] S.R. Dhage, V. Ravi, S.K. Date, Bullet. Mater. Sci. 27 (2004) 43.

[9] J.F. McAleer, P.T. Moseley, J.O.W. Norris, D.E. Williams, J. Chem. Soc. Farad. Trans. 183 (1987) 1323.

[10] R. Ionescu, A Vancu, C.-C. Moise, A Tomescu, Sensors and Actuators B 61 (1999) 39.

[11] P.G. Harrison, M.J. Willett, J. Chem. Soc. Farad. Trans. 185 (1989) 1921.

[12] G. Korotchenko, V. Brynzari, S. Dmitriev, Sensors and Actuators B 107 (2005) 209.

[13] M. Nakagawa, H. Mitsuo, Surf. Sci. 175 (1986) 157.

[14] V. Lantto, Gas Sensors (Springer, Netherlands, 1992).

[15] S. Ahlers, G. Muller, T. Doll, Sensors and Actuators B 107 (2005) 587.

[16] M.V. Kalinina, V.A. Moshnikov, P. Tikhonov, V. Tomaev, S.V. Mikhailichenko, Glass Phys. Chem. 29 (2003) 422.

[17] Y.T. Jang, S.I. Moon, J.H. Ahn, Y.H. Lee, B.K. Ju, Sensors and Actuators B 99 (2004) 118. 
[18] V.M. Aroutiounian, A.Z. Adamyan, E.A. Khachaturyan, Z.N. Adamyan, K. Hernadi, Z. Pallai, Z. Nemeth, L. Forro, A. Magrez, E. Horvath, Sensors and Actuators B 177 (2013) 308.

[19] Z.N. Adamyan, A.G. Sayunts, E.A. Khachaturyan, V.M. Aroutiounian, Journal of Contemporary Physics (Armenian Academy of Sciences) 51 (2016) 143.

[20] V.I. Gaman, Russian Phys. J. 54 (2012) 1364.

[21] G.Di Francia, B. Alfano, V.La Ferrara, J. Sensors 29 (2009) 18.

[22] K.B. Karandeev, Special Methods of Electrical Measurements (Gosenergoizdat, Moscow, 1963). 